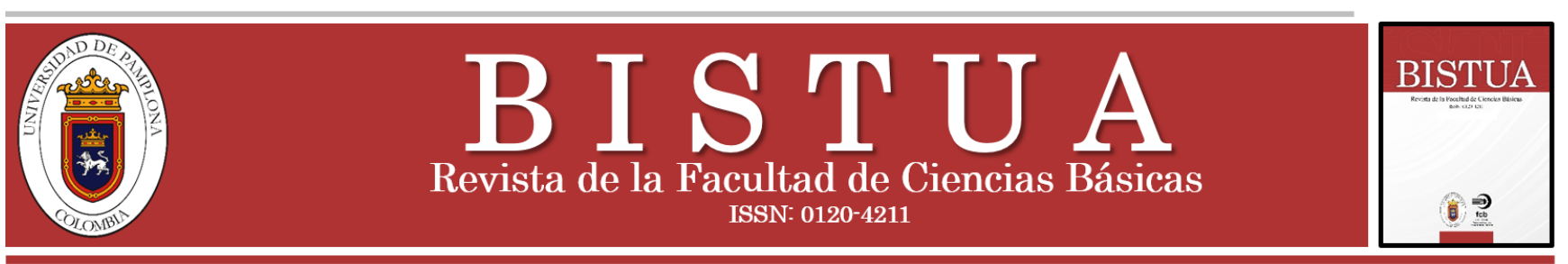

http://revistas.unipamplona.edu.co/ojs_viceinves/index.php/BISTUA

\title{
Modelos de ecuaciones estructurales aplicados al análisis de la evaluación del aprendizaje sobre el impacto del ruido
}

\section{Models of structural equations applied to the analysis of the evaluation of learning on the impact of noise}

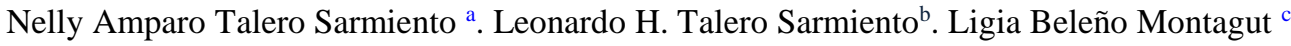 \\ ${ }^{a}$ Instituto Integrado Francisco Serrano Muñoz sede A san Juan de Girón, Colombia. \\ ${ }^{b}$ Escuela de estudios Industriales y Empresariales, Universidad industrial de Santander, Colombia. \\ ${ }^{c}$ Departamento de matemáticas y ciencias naturales, Universidad Autónoma de Bucaramanga, Colombia. \\ Contacto:lbeleno@unab.edu.co
}

Recibido: Noviembre 24, 2020. Diciembre 18, 2020

https://doi.org/10.24054/01204211.v2.n2.2020.4439

\section{Resumen}

Es importante conocer cómo el ruido afecta tanto la salud como las experiencias de aprendizaje. Lo anterior toma relevancia particularmente en instituciones de educación básica y media, ya que éstas suelen estar ubicadas en cabeceras municipales. El presente proyecto busca determinar si una propuesta pedagógica multidisciplinaria puede fortalecer el conocimiento en temas como Sonido, Ruido, y el impacto del Ruido en la salud. Para ello se propone un estudio longitudinal evaluando los conocimientos previos y posteriores a la aplicación de cuatro unidades didácticas: 1) Socialización, 2) Exposición oral, 3) Desarrollo de folletos y, 4) Investigación dirigida. Para determinar si la propuesta es significativa, se desarrollan diferentes modelos de ecuaciones estructurales los cuales son analizados a luz de sus ajustes estadísticos, y la significancia de sus parámetros; los resultados del presente trabajo implican que los estudiantes luego de la aplicación de las cuatro unidades didácticas aumentaron su conocimiento en cuanto a los conceptos de Ruido y su impacto en la salud; no obstante, hay aspectos a mejorar en cuanto al conocimiento sobre el Sonido y su diferencia con el Ruido.

Palabras clave: Aprendizaje; Contaminación Sonora; Modelo estadístico; Pedagogía, Ruido.

\section{Introducción}

El sonido, es la propagación de ondas mecánicas que vibran en un medio elástico (aire, líquido y sólido), transversales y longitudinales en sólidos, pero solo longitudinales en líquidos y gases, el cual se empieza a percibir como ruido cuando es molesto para el oído humano; la generación simultánea de ruidos desde diferentes fuentes se conoce como contaminación sonora [1]. En el contexto colombiano, se establece la norma nacional de emisión de ruido y ruido ambiental, en la cual se definen los parámetros, unidad de

\section{Abstract}

It is important to know how noise affects both health and learning experiences. This is particularly relevant in basic and secondary education institutions, since these are usually located in municipal capitals. This project seeks to determine if a multidisciplinary pedagogical proposal can strengthen knowledge on topics such as Sound, Noise, and the impact of Noise on health. For this, a longitudinal study is proposed evaluating the knowledge before and after the application of four didactic units: 1) Socialization, 2) Oral presentation, 3) Development of brochures and, 4) Directed research. To determine if the proposal is significant, different models of structural equations are developed which are analyzed in light of their statistical adjustments, and the significance of their parameters; The results of this work imply that the students after the application of the four didactic units increased their knowledge regarding the concepts of Noise and its impact on health; however, there are aspects to improve in terms of knowledge about Sound and its difference with Noise.

Keywords: Learning; Noise pollution; Statistical model; Pedagogy; Noise medida, niveles de intensidad, tiempos de exposición, horarios, y estándares Máximos Permisibles de Niveles de Ruido Ambiental [2]. En este documento se presenta un proceso de investigación de carácter formativo desde el aula de clase, enfocado particularmente en la apropiación de los siguientes conceptos (o constructos).

- Conocimientos generales sobre el sonido

- Conocimiento general sobre la contaminación acústica

- Conocimiento general sobre la relación entre el ruido y la salud. 
El ruido es un factor que incide negativamente en la calidad de vidas de las personas, particularmente en aquellas que viven en ciudades [3]. Particularmente, se encuentra que existen diferentes fuentes de contaminación acústica en los centros escolares, las externas (ruidos generados por la circulación del tránsito y las actividades de comercio de los alrededores) y las internas (generadas por los gritos de los estudiantes y actividades prácticas propias escolares), estas fuentes se caracterizan no solamente por afectar la salud, sino adicionalmente el proceso de enseñanza-aprendizaje [4], ya que éste disminuye la capacidad de escucha y atención de los jóvenes en los colegios [5], por consiguiente, es necesario concientizar a los estudiantes sobre el efecto del ruido en la salud.

Bajo esta premisa, se encuentran que diferentes instituciones han realizado trabajos en relación al Sonido, Ruido, y su Impacto en la salud, ya que no todo sonido es ruido, y dependiendo de la exposición y el nivel del mismo (en decibeles) puede afectar a las personas [6]. Entre los trabajos desarrollados se encuentra que el conocimiento sobre el ruido se suele medir mediante en escalas ordenadas, basándose particularmente en la percepción o conocimiento sobre diferentes conceptos [7,8]; ya que existe dificultades para evaluar el impacto en la salud, y para ello se requiere de mediciones en el sitio de trabajo y hacer un seguimiento a las intensidades sonoras [9].

El presente trabajo se alinea con la literatura al proponer un estudio enfocado en la importancia del conocimiento sobre el impacto del ruido en la salud delimitado al aula de clases en estudiantes de colegio. Para ello se desarrolla un estudio longitudinal a estudiantes de grados noveno, décimo, y undécimo; luego de un análisis de conocimientos previos se les aplican diferentes actividades pedagógicas o secuencias didácticas que incluyen la lectura colaborativa acerca de los conceptos de sonido, ruido, fuentes, escalas de medición, y efectos en la salud. Posteriormente, se desarrolla una conceptualización guiada la cual incluye el diligenciamiento de una guía, consecutivamente y como actividad en clase se diseñan y construyen folletos que incluyen mapas mentales para la socialización a nivel Colegio mediante una campaña escolar. Como actividad final, se desarrolla un proyecto de fin de periodo en el cual los alumnos haciendo uso de aplicativos móviles realizan un mapa de ruido del Colegio. Una vez realizadas las actividades, se aplica un segundo instrumento estadístico para determinar el efecto de las secuencias didácticas en el aprendizaje sobre el impacto del ruido en la salud de los estudiantes.

Con el fin de determinar la posible apropiación, se propone una estrategia de enseñanza aprendizaje que parte de un estudio exploratorio con el fin de evaluar los preconceptos asociados a los temas de interés. Una vez finalizada esta etapa, durante el periodo académico se realizarán diferentes actividades siguiendo unas secuencias didácticas; tras la finalización de las mismas se aplica un segundo instrumento estadístico con el fin de evaluar la apropiación conceptual.
En este sentido, el presente trabajo propone las siguientes hipótesis de investigación.

$H_{0}^{1}$ El conocimiento sobre los constructos no depende de la propuesta pedagógica, es decir, una vez se apliquen las experiencias de enseñanza-aprendizaje, la valoración de conocimiento en los tres constructos no aumentará.

$H_{0}^{2}$ El modelo exploratorio de ecuaciones estructurales tiene un ajuste significativo, lo cual implica que los estudiantes tienen un claro conocimiento previo interrelacionado sobre los constructos.

$H_{0}^{3} \mathrm{El}$ modelo evaluativo de ecuaciones estructurales tiene un ajuste significativo, lo que implica que los estudiantes tienen un claro conocimiento final interrelacionado sobre los constructos.

\section{Método}

El presente estudio se realizó en un colegio oficial de Santander (Colombia), durante el año 2017. A todos los estudiantes que se encontraban cursando entre noveno a undécimo grado de la sede A en la jornada de la tarde, conformando así una población reducida. Los análisis exploratorios y evaluativos se realizaron a todos los individuos de la población. En la aplicación de la encuesta no se encontraron datos perdidos ni respuestas anuladas, con lo cual se obtiene una base de datos de 28 columnas conformadas por las 12 preguntas relacionadas con los constructos bajo estudio en un estudio exploratorio, 12 preguntas relacionadas con los constructos bajo estudio en un estudio evaluativo, y variables asociadas a las características de los estudiantes: Edad, Grado, Curso, y Género; y un total de 181 registros asociados a los estudiantes partícipes del proyecto.

Tabla 1. Estadísticos descriptivos asociados a las variables analizadas

\begin{tabular}{|c|c|c|c|c|c|c|c|c|c|c|}
\hline Variable & $N$ & Valor ${ }^{M}$ & $\begin{array}{l}\text { edia } \\
\text { Error std }\end{array}$ & Dessu Esst & Minimo & $Q 1$ & Mediana & $Q$ & Móximo & \\
\hline Edad & 181 & 15,718 & 0,0844 & 1,179 & 13 & 15 & 16 & 17 & 18 & \\
\hline Grado & 181 & & & & 9 & 9 & 10 & 11 & 11 & \\
\hline Curso & 181 & & & & 3 & 3 & 3 & 4 & 4 & \\
\hline P1 & 181 & 3,1602 & 0,0503 & 0,6764 & 1 & 3 & 3 & 4 & 5 & \\
\hline P2 & 181 & 3,1271 & 0,0897 & 1,2066 & 1 & 2 & 3 & 4 & 5 & \\
\hline P3 & 181 & 3,6022 & 0,0673 & 0,9048 & 1 & 3 & 4 & 4 & 5 & \\
\hline P4 & 181 & 3,4199 & 0,091 & 1,2249 & 1 & 2 & 4 & 4 & 5 & चี \\
\hline P5 & 181 & 4,1934 & 0,0897 & 1,207 & 1 & 4 & 5 & 5 & 5 & \\
\hline P6 & 181 & 4,0497 & 0,0957 & 1,2879 & 1 & 4 & 5 & 5 & 5 & \\
\hline P7 & 181 & 4,0663 & 0,0505 & 0,6799 & 2 & 4 & 4 & 5 & 5 & 芴 \\
\hline P8 & 181 & 4,0829 & 0,0775 & 1,0429 & 1 & 4 & 4 & 5 & 5 & ్ㅡㅁ \\
\hline P9 & 181 & 3,3591 & 0,0631 & 0,8487 & 1 & 3 & 3 & 4 & 5 & \\
\hline P10 & 181 & 4,0829 & 0,0655 & 0,8812 & 1 & 4 & 4 & 5 & 5 & \\
\hline P11 & 181 & 4,0055 & 0,0737 & 0,9916 & 1 & 4 & 4 & 5 & 5 & \\
\hline P12 & 181 & 4,2873 & 0,0766 & 1,0303 & 1 & 4 & 5 & 5 & 5 & \\
\hline P13 & 181 & 3,4144 & 0,0422 & 0,5673 & 3 & 3 & 3 & 4 & 5 & \\
\hline P14 & 181 & 3,4365 & 0,0472 & 0,6347 & 3 & 3 & 3 & 4 & 5 & \\
\hline P15 & 181 & 4,1768 & 0,0361 & 0,485 & 3 & 4 & 4 & 4 & 5 & 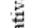 \\
\hline P16 & 181 & 4,2707 & 0,0383 & 0,515 & 3 & 4 & 4 & 5 & 5 & 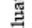 \\
\hline P17 & 181 & 4,4365 & 0,0378 & 0,5084 & 3 & 4 & 4 & 5 & 5 & 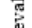 \\
\hline P18 & 181 & 4,6077 & 0,0372 & 0,5008 & 3 & 4 & 5 & 5 & 5 & \\
\hline P19 & 181 & 4,6133 & 0,0363 & 0,4884 & 4 & 4 & 5 & 5 & 5 & 要 \\
\hline P20 & 181 & 4,326 & 0,0358 & 0,4817 & 3 & 4 & 4 & 5 & 5 & \\
\hline P21 & 181 & 4,4641 & 0,0403 & 0,5427 & 3 & 4 & 4 & 5 & 5 & \\
\hline
\end{tabular}

En la Tabla 1 se registran los estadísticos descriptivos de las variables bajo análisis, se resalta que, para el caso de Grado y Curso, no se registran tendencias centrales ni dispersión ya que son variables nominales. Por otra parte, en la Fig. 1 se presenta la matriz de correlación entre las 
variables donde se aprecia una tendencia diferente para cada subconjunto de preguntas (de la pregunta 1 a la 12, y de la 13 a la 24).

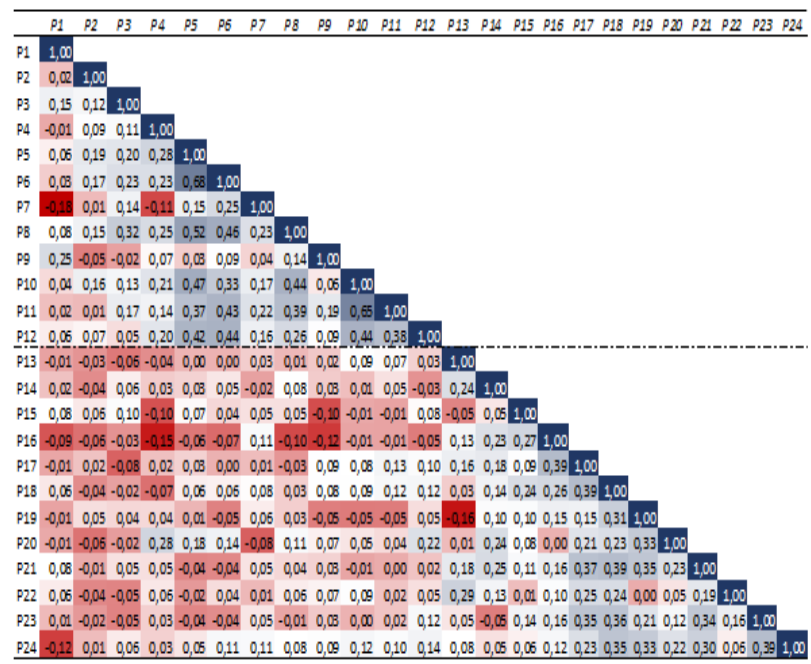

Figura 1. Matriz de correlación entre las variables bajo análisis Fuente: autor(es).

Por otra parte, para determinar la consistencia del instrumento se calcula el alfa de Cronbach., el cual si presenta valores superiores a 0.6 se puede considerar que el instrumento es consistente y adecuado [10], en este caso es posible es posible agrupar preguntas (variables) mediante técnicas estadísticas multivariantes siempre y cuando el índice de adecuación KMO sea superior a 0.50, la prueba de esfericidad de Bartlette sea estadísticamente significativa ( $p$ $<0.05$ ) [11]. En la Tabla 2 se registran los valores obtenidos para las pruebas e índices; teniendo en cuenta dichos valores, es posible contrastar diferentes modelos de ecuaciones estructurales con el fin de evaluar las hipótesis presentadas previamente [12].

Tabla 2. Valores obtenidos para las pruebas e índices

\begin{tabular}{llll}
\hline Constructo & KMO & EB & Alpha \\
\hline Dataset exploratorio & 0,74 & 0,00 & 0,75 \\
Dataset evaluativo & 0,73 & 0,00 & 0,72 \\
\hline
\end{tabular}

\section{Fase exploratoria}

Posterior al desarrollo de las actividades, se evidenció el logro en el aprendizaje acerca de la diferencia entre sonido y ruido, y como paso siguiente la relación que tienen dichos conceptos con los efectos sobre la salud.

Por lo anterior, se planteó el modelo de ecuaciones estructurales, con el fin de representar la interacción entre los constructos propuestos y su modelo de relación (influencia del conocimiento sobre sonido y conocimiento sobre ruido en el conocimiento sobre la afectación del ruido en la salud) mediante dos escenarios: En el primero, cada una de las preguntas explica el constructo para el cual fue diseñado (Fig. 2) alrededor de los tópicos mencionados anteriormente, y en el segundo, el constructo Conocimiento sobre el Sonido y
Conocimiento sobre ruido, y cómo influyen en el Conocimiento sobre el efecto del ruido en la salud (Fig. 3).
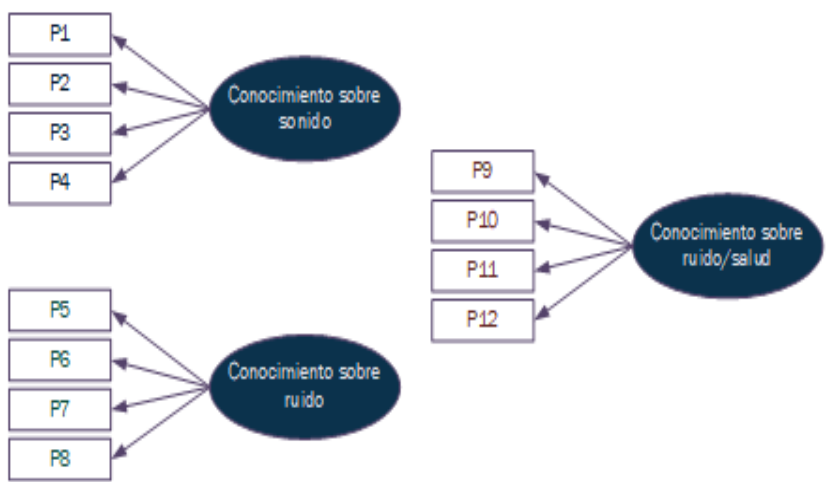

Figura 2. Modelo de Análisis Factorial Confirmatorio para la etapa exploratoria

Fuente: autor(es).

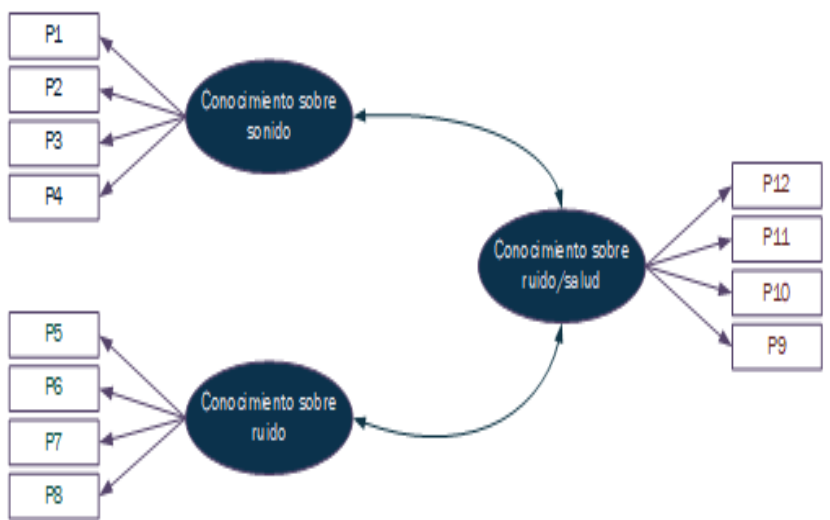

Figura 3. Modelo de ecuaciones estructurales para la etapa exploratoria Fuente: autor(es).

Partiendo del supuesto de que los estudiantes no reconocen la contaminación acústica como un problema en el entorno escolar e ignoran sus efectos en la salud de los miembros de la comunidad educativa, se diseñaron una serie de actividades y unidades didácticas que se aplicaron en los estudiantes como estrategia para cumplir con los objetivos propuestos utilizando el modelo de secuencia didáctica. En esta estrategia se involucraron directamente los estudiantes desde grado noveno hasta grado undécimo, objeto de estudio de la investigación, aunque en algunas de las actividades diseñadas se realizó una proyección y socialización de la problemática a los demás estudiantes de la comunidad educativa.

El diseño de las unidades didácticas de la secuencia se realizó con el objetivo de poder abarcar los tres constructos principales de la investigación (conocimientos generales sobre sonido, contaminación acústica y relación entre ruido y salud), buscando integrar diversas áreas (matemáticas, física, artística, ética, ciencias naturales, etc.), apoyándose en un proyecto transversal como lo es la educación ambiental, de tal forma que se genere mayor motivación para el desarrollo de las mismas y se logre un aprendizaje significativo en los estudiantes. La estructura de la propuesta pedagógica se 
observa en la Fig. 4 y a continuación, se describen los detalles de cada una de las fases.

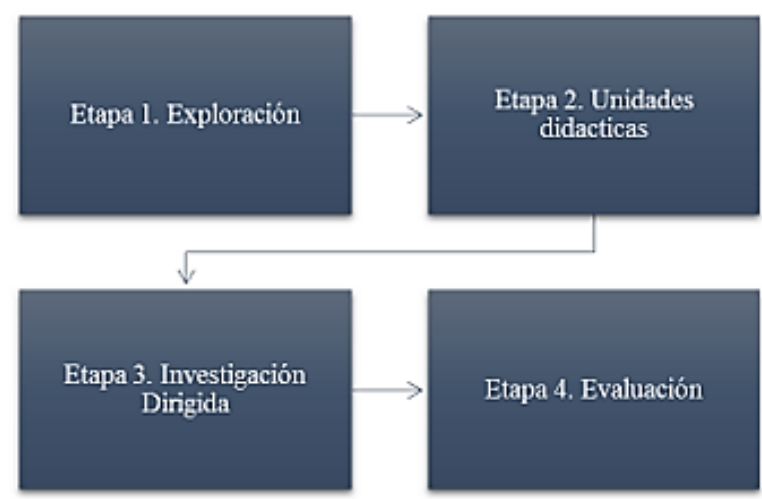

Figura 4. Etapas de la propuesta pedagógica Fuente: autor(es).

Para la etapa de exploración Se realiza un taller de lectura (Incluido dentro del Plan Lector Institucional) a todos los estudiantes desde grado noveno. Durante esta etapa es diseñada una serie de talleres sobre el tema de sonido, ruido, y contaminación auditiva con una duración de una hora. Los talleres son implementados dentro del Plan Escolar de Lectura, proyecto en el que todas las áreas deben diseñar y aplicar talleres de lectura, escritura y oralidad sobre diversos temas. Se eligió esta modalidad de taller con el fin de que todos los estudiantes se encontraran realizando las actividades de forma simultánea. Así mismo, se seleccionaron estudiantes de grado once que lideraron los procesos en cada una de las aulas, de tal forma que pudieran solucionar las dudas o dificultades que se pudieran presentar.

Se diseña una propuesta de investigación sobre la problemática de contaminación acústica en cada uno de los niveles de noveno a once mediante la propuesta de proyecto de aula; permitiendo que: primero, los estudiantes consultaran sobre el tema; segundo, realizaran mediciones de ruido en diferentes zonas de la institución y; tercero, elaboraran una campaña escolar sobre sensibilización en los diferentes cursos. Finalmente, se realiza una sustentación oral sobre el trabajo realizado ante sus compañeros de clase. La propuesta se diseñó para ser elaborada en cada una de las áreas en la cual la docente era titular y acorde al nivel de cada grado.

La evaluación de la propuesta pedagógica se hizo realizando a la aplicación de cada una de las fases descritas. Cada actividad propuesta tuvo una nota cuantitativa dentro del plan de trabajo de los estudiantes para cada una de las áreas descritas. De igual forma, se diseñó una rejilla de evaluación para valorar el proyecto de aula que incluía una serie de ítems relacionados con las fases y actividades que debían realizar, además de la autoevaluación, coevaluación y heteroevaluación.

\section{Resultados}

Con el fin de identificar si los objetos de aprendizaje aplicados durante el curso académico generaron una diferencia respecto a los conocimientos sobre sonido, ruido y sus implicaciones en la salud, durante la segunda etapa del presente trabajo se formulan dos variantes al modelo de ecuaciones estructurales:

- Variante 1: Modelo sin depender del modelo exploratorio; con esta variante se analiza si el conocimiento en la etapa evaluativa es explicado mediante los constructos formulados.

- Variante 2: Modelo con dependencia del modelo exploratorio; con esta variante se busca determinar si el conocimiento referente a los constructos propuestos depende del conocimiento evaluado en la etapa exploratoria.

De manera general, el modelo propuesto durante el presente trabajo pasa la prueba de bondad de ajuste (Chisquare, Chi cuadrado $X^{2}$ ), indicando que se ha logrado ajustar a las variables observadas, latentes y sus relaciones, a la par de obtener buenas calificaciones en dos estadísticos denominados CFI y NNFI. El primero compara el estadístico Chi-square (mediante una acotación entre 0 y 1 , donde el 1 representa un ajuste perfecto) al comparar la discrepancia entre la matriz de covarianzas que predice el modelo y la matriz de covarianzas observada, mientras que el NNFI funciona de manera similar, corregido para tener en cuenta la complejidad del modelo. Por otra parte, al observar los valores de pendiente y su significancia estadística sumado a que valores altos en la escala de Likert indican mayor conocimiento, se puede determinar que las preguntas pertenecientes al constructo Conocimiento del efecto del ruido en la salud, son las más sensibles al valor de las respuestas por parte de los estudiantes, lo anterior puede indicar que cuando los estudiantes contestan indicando que conocen del tema, el constructo toma mayor fuerza, para finalizar, se evidencia la relación estadísticamente significativa entre los constructos, por tanto, se determina que no existe suficiente información estadística para rechazar la Hipótesis nula 2, es decir, el modelo propuesto de ecuaciones estructurales logra representar el conocimiento presentado por los estudiantes sobre sonido, ruido y sus implicaciones en la salud. Los resultados de ajuste del modelo estructural de medida se muestran en la Tabla 3.

Tabla 3. Resultados de ajuste del modelo estructural de medida

\begin{tabular}{cccr}
\hline $\begin{array}{c}\text { Indice de } \\
\text { ajuste }\end{array}$ & $\begin{array}{c}\text { Recomenda } \\
\text { do }\end{array}$ & $\begin{array}{c}\text { Valor } \\
\text { del modelo }\end{array}$ & $\begin{array}{r}\text { Ajuste } \\
\text { del modelo }\end{array}$ \\
\hline $\begin{array}{c}\text { Chi- } \\
\text { square }\end{array}$ & $\mathrm{p} \geq 0,05$ & $\begin{array}{c}43,55 \\
(\mathrm{p}=0,761)\end{array}$ & Bueno \\
\hline $\begin{array}{c}\text { NNFI } \\
\text { (Non-normed } \\
\text { fit index) }\end{array}$ & $\geq 0,8$ & 1,000 & Bueno \\
\hline $\begin{array}{c}\text { CFI } \\
\text { (Comparativ } \\
\text { e fit index) }\end{array}$ & $\geq 0,8$ & 1,000 & Bueno \\
\hline
\end{tabular}




\section{Discusión}

Una vez aplicadas las estrategias de enseñanzaaprendizaje, se realiza el componente evaluativo. El valor de Chi cuadrado es inferior a 0.05 , lo cual indica un ajuste deficiente; no obstante, los otros criterios sí indican un buen modelo. Por otra parte, se evidencia que las variables observadas relacionadas con el conocimiento sobre el sonido presentan un bajo ajuste, es decir, no se encuentra una relación estadísticamente significativa respecto a este constructo. Para finalizar, se encuentra que sólo se cumple la relación de Conocimiento del Ruido - Conocimiento del efecto del Ruido, es decir, el conocimiento sobre el ruido permite entender su efecto sobre la salud humana.

Por otra parte, respecto a la variante dos se encuentra que el modelo no presenta un buen ajuste, este fenómeno se evidencia en la cantidad de elementos que no presentan un ajuste significativo lo que indica que la segunda variante no es adecuada y, por tanto, el conocimiento previo no incide sobre el conocimiento tras la experiencia propuesta pedagógica. Lo anterior se puede proponer ya que dentro de las pendientes que indican poca o ninguna relación entre constructos se encuentran: Conocimiento sobre sonido Antes con pendiente $-0,101$ y $p$ valor de 0,673 respecto al Conocimiento sobre sonido Después, Conocimiento sobre ruido Antes con pendiente 0,195 y p valor de 0,515 respecto al Conocimiento sobre ruido Después y, finalmente, Conocimiento sobre afectación Antes con pendiente 0,056 y $\mathrm{p}$ valor de 0,240 respecto al Conocimiento sobre afectación Después. En añadidura, se determina que no existe completa claridad sobre el conocimiento relacionado con el sonido en la etapa evaluativa (después) al menos con una confiabilidad del $95 \%$, debido a los p valores cercanos a 0.08 de cada pregunta respecto a su constructo.

Síntesis de las hipótesis:

Hipótesis Nula 1- No aceptada

Los modelos de ecuaciones estructurales exploratorio (modelo en la etapa 1) y evaluativo (modelo en la etapa 2, posterior a la aplicación de la propuesta pedagógica) poseen ajuste significativo y presentan diferencias de media y variabilidad en sus preguntas y constructos. Además, no existe suficiente información estadística que indique que esa diferencia depende de un conocimiento previo que influye en el nuevo, lo que da cabida a interpretar que la propuesta pedagógica de aprendizaje influyó significativamente en el cambio

Hipótesis Nula 2- No rechazada

A partir de la estimación de los parámetros de ajuste se determina que el modelo de ecuaciones estructurales representa la interacción entre los constructos propuestos y su modelo de relación (influencia del conocimiento sobre sonido y conocimiento sobre ruido en el conocimiento sobre la afectación del ruido en la salud).
Hipótesis Nula 3- No rechazada

A partir de la estimación de los parámetros de ajuste se determina que, si bien no posee un buen valor de chi cuadrado, el modelo de ecuaciones estructurales representa la interacción entre los constructos propuestos y su modelo de relación (influencia del conocimiento sobre sonido y conocimiento sobre ruido en el conocimiento sobre la afectación del ruido en la salud). El valor de Chi cuadrado es susceptible a la cantidad de relaciones en el modelo y, por tanto, es posible concluir el ajuste basado en los estadísticos NNFI y CFI.

\section{Conclusiones}

El uso del cuestionario sobre Conocimientos de Sonido, ruido y efectos del ruido en la salud (Ver Anexos), permitió diagnosticar los conocimientos previos que tenían los estudiantes sobre los aspectos conceptuales relacionados con la Contaminación Acústica, permitiendo identificar que, los estudiantes no poseen suficiente conocimiento con respecto al sonido teniendo en cuenta que los porcentajes estuvieron entre el $40 \%$ y $59 \%$, y los resultados muestran que pueden confundir un sonido alto con el ruido. Las cuatro preguntas relacionadas con el constructo de Conocimientos sobre Ruido tuvieron porcentajes que se encontraron en el rango del $81 \%$ a $83 \%$, lo cual implica que reconocen esa problemática en su entorno escolar, pero no lo relacionan con los efectos que tiene el ruido en la salud de las personas, al encontrarse en las respuestas mayor variación en el tercer constructo relacionado con los efectos del ruido en la salud cuyos porcentajes se encontraron en el rango del $44 \%$ al $85 \%$.

El presente trabajo de investigación se estructuró con el fin de determinar si una propuesta pedagógica incide sobre la apropiación de conocimientos relacionados con el sonido, ruido y su impacto en la salud. Para ello y según las aproximaciones de los trabajos realizados por $[7,8]$ se desarrolló un modelo de ecuaciones estructurales haciendo uso de encuestas auto suministradas con variables en escala Likert. Los resultados permiten determinar que la experiencia académica desarrollada tuvo un impacto positivo en el proceso de aprendizaje; no obstante, se identifican aspectos de mejora respecto al concepto de Sonido, lo cual puede deberse a que su definición está fuertemente articulada con la de Ruido y, para la edad de los participantes (estudiantes entre 13 a 18 años), ciertos conceptos pueden ser difusos. Por tanto, se recomienda la implementación de metodologías afines en otros niveles de formación, es decir, desarrollar trabajos de corte longitudinal que permitan determinar la apropiación conceptual de diversos temas, lo anterior con el fin de hacer un seguimiento más detallado al proceso formativo de los alumnos. 


\section{Referencias}

[1] Tezel, M. N., Sari, D., Ozkurt, N., \& Keskin, S. S. Combined NOx and noise pollution from road traffic in Trabzon, Turkey. Science of The Total Environment, 696, (2019) 134044. https://doi.org/10.1016/j.scitotenv.2019.134044

[2] Resolución Número (627), 29 (testimony of MinAmbiente) (2006)

[3] Silva, L. T., Oliveira, I. S., \& Silva, J. F. The impact of urban noise on primary schools. Perceptive evaluation and objective assessment. Applied Acoustics, 106, (2016). 2-9. https://doi.org/10.1016/j.apacoust.2015.12.013

[4] Connolly, D. M., Dockrell, J. E., Shield, B. M., Conetta, R., \& Cox, T. J.. Students' Perceptions of School Acoustics and the Impact of Noise on Teaching and Learning in Secondary Schools: Findings of a Questionnaire Survey. Energy Procedia, 78, (2015) 3114-3119. https://doi.org/10.1016/i.egypro.2015.11.766

[5] Santika, B. B., Indrawati, S., Suyatno, \& Yahya, E. Noise Evaluation of Traffic Flows and Its Effect to Concentration Capability of the Students in One of Private School in Surabaya. Procedia Engineering, 170, (2017). 274-279. https://doi.org/10.1016/j.proeng.2017.03.026

[6] Dzhambov, A., Tilov, B., Markevych, I., \& Dimitrova, D. Residential road traffic noise and general mental health in youth: The role of noise annoyance, neighborhood restorative quality, physical activity, and social cohesion as potential mediators. Environment International, 109, . (2017), 1-9. https://doi.org/10.1016/j.envint.2017.09.009

[7] Franca, M. C. A comparison of vocal demands with vocal performance among classroom student teachers. Journal of Communication Disorders, 46(1), (2013). 111-123. https://doi.org/10.1016/j.jcomdis.2012.11.001

[8] Wen, X., Lu, G., Lv, K., Jin, M., Shi, X., Lu, F., \& Zhao, D. Impacts of traffic noise on roadside secondary schools in a prototype large Chinese city. Applied Acoustics, 151, (2019). 153-163. https://doi.org/10.1016/j.apacoust.2019.02.024

[9] Huertas Bustos, A. P., Vesga Bravo, G. J., \& Galindo León, M.. Validación del instrumento 'inventario de habilidades metacognitivas (Mai)' con estudiantes colombianos. Praxis \& Saber, 5(10), (2014) 55. https://doi.org/10.19053/22160159.3022

[10] Munier, C., Brockmann-Bauser, M., Laukkanen, A.-M., Ilomäki, I., Kankare, E., \& Geneid, A. Relationship Between Laryngeal Signs and Symptoms, Acoustic Measures, and Quality of Life in Finnish Primary and Kindergarten School Teachers. Journal of Voice, 34(2), (2020). 259-271. https://doi.org/10.1016/j.jvoice.2018.12.006

[11] Scherer, R., Tondeur, J., Siddiq, F., \& Baran, E. The importance of attitudes toward technology for pre-service teachers' technological, pedagogical, and content knowledge: Comparing structural equation modeling approaches. Computers in Human Behavior, 80, (2018). 6780. https://doi.org/10.1016/j.chb.2017.11.003

[12] Gil-Madrona, P., Méndez Hinojosa, L. M., Pérez-Segura, J. J., SáezSánchez, M. B., \& Zamora Poblete, G. Scale of Pedagogical Authority Meanings in the classroom (ESAPA) for Ibero-America built on the opinions of teaching students. Teaching and Teacher Education, 93, (2020).103079. https://doi.org/10.1016/j.tate.2020.103079 


\section{Anexos}

Anexo A Preguntas del instrumento exploratorio

La siguiente encuesta hace parte de un estudio de investigación. Está compuesta por preguntas destinadas a conocer sus saberes y opiniones sobre el sonido y ruido. Las preguntas tienen una escala de uno a cinco, (1 corresponde al menor grado y 5 al mayor grado) de la cual solamente debe seleccionar una opción marcando con una x sobre la respuesta que usted considere según sea el caso.

$\begin{array}{lll}\text { EDAD: } & \text { GÉNERO: } & \text { Femenino_ } \\ \text { Masculino__ } & \text { GRADO: } & \end{array}$

¿Qué tanto conocimiento tiene sobre el sonido?

1. Ninguno

2. Poco

3. Básico

4. Suficiente

5. Mucho

II. ¿Está de acuerdo en que el sonido se puede propagar en el vacío (espacio exterior)?

1. Totalmente en desacuerdo

2. En desacuerdo

3. Indiferente (ni en acuerdo ni en desacuerdo)

4. De acuerdo

5. Totalmente de acuerdo

III. ¿Está de acuerdo en que un sonido agudo tiene una alta frecuencia?

1. Totalmente en desacuerdo

2. En desacuerdo

3. Indiferente (ni en acuerdo ni en desacuerdo)

4. De acuerdo

5. Totalmente de acuerdo

IV. ¿Está de acuerdo en que el oído humano solo puede escuchar un rango de sonidos específicos?

1. Totalmente en desacuerdo

2. En desacuerdo

3. Indiferente (ni en acuerdo ni en desacuerdo)

4. De acuerdo

5. Totalmente de acuerdo

V. ¿Está de acuerdo en que el exceso de ruido es un tipo de contaminación?

1. Totalmente en desacuerdo

2. En desacuerdo

3. Indiferente (ni en acuerdo ni en desacuerdo)

4. De acuerdo

5. Totalmente de acuerdo

VI. ¿Está de acuerdo que dentro del colegio se generan altos niveles de ruido?

1. Totalmente en desacuerdo

2. En desacuerdo

3. Indiferente (ni en acuerdo ni en desacuerdo)

4. De acuerdo

5. Totalmente de acuerdo

VII. Considera que el ruido generado dentro de la institución es:

1. $\quad$ Nulo (no se genera ruido)

2. Débil

3. Moderado

4. Fuerte

5. Intolerable

VIII. ¿Está de acuerdo que por fuera del colegio se generan altos niveles de ruido? Como por ejemplo ruido por vehículos, etc.

1. Totalmente en desacuerdo

2. En desacuerdo

3. Indiferente (ni en acuerdo ni en desacuerdo)

4. De acuerdo

5. Totalmente de acuerdo

IX. ¿Qué tanto conocimiento tiene sobre el daño que puede causar el ruido en la salud las personas?

1. Ninguno

2. Poco
3. Básico

4. Suficiente

5. Mucho

X. ¿Está usted de acuerdo en que la exposición prolongada al ruido puede generar estrés en las personas?

1. Totalmente en desacuerdo

2. En desacuerdo

3. Indiferente (ni en acuerdo ni en desacuerdo)

4. De acuerdo

5. Totalmente de acuerdo

XI. ¿Está usted de acuerdo en que la exposición prolongada al ruido puede generar pérdida de audición en las personas?

1. Totalmente en desacuerdo

2. En desacuerdo

3. Indiferente (ni en acuerdo ni en desacuerdo)

4. De acuerdo

5. Totalmente de acuerdo

XII. ¿Está usted de acuerdo en que el ruido disminuye la concentración durante las clases?

1. Totalmente en desacuerdo

2. En desacuerdo

3. Indiferente (ni en acuerdo ni en desacuerdo)

4. De acuerdo

5. Totalmente de acuerdo

Gracias por su colaboración. 
Anexo B. Preguntas del instrumento evaluativo.

La siguiente encuesta hace parte de un estudio de investigación. Está compuesta por preguntas destinadas a conocer sus saberes y opiniones sobre el sonido y ruido después de haber realizado el proyecto sobre sonido y ruido en la Institución Educativa. . Las preguntas tienen una escala de uno a cinco, ( 1 corresponde al menor grado y 5 al mayor grado) de la cual solamente debe seleccionar una opción marcando con una x sobre la respuesta que usted considere según sea el caso.

EDAD: GRADO:

GÉNERO: Femenino__ Masculino_

I. ¿Qué nivel de conocimiento tiene sobre el sonido?

1. Nada

2. Poco

3. Medio

4. Bastante

5. Mucho

II. ¿En qué medida comprende que las ondas sonoras necesitan un medio poder propagarse?

1. Nada

2. Poco

3. Medio

4. Bastante

5. Mucho

III. ¿Está de acuerdo en que un sonido agudo tiene una alta frecuencia?

1. Totalmente en desacuerdo

2. En desacuerdo

3. Indiferente (ni en acuerdo ni en desacuerdo)

4. De acuerdo

5. Totalmente de acuerdo

IV. ¿Está de acuerdo en que el oído humano solo puede escuchar un rango de sonidos específicos?

1. Totalmente en desacuerdo

2. En desacuerdo

3. Indiferente (ni en acuerdo ni en desacuerdo)

4. De acuerdo

5. Totalmente de acuerdo

V. ¿Está de acuerdo en que el ruido es cualquier sonido que llega a ser molesto para el oído humano?

1. Totalmente en desacuerdo

2. En desacuerdo

3. Indiferente (ni en acuerdo ni en desacuerdo)

4. De acuerdo

5. Totalmente de acuerdo

VI. ¿Está de acuerdo en que el ruido es un contaminante?

1. Totalmente en desacuerdo

2. En desacuerdo

3. Indiferente (ni en acuerdo ni en desacuerdo)

4. De acuerdo

5. Totalmente de acuerdo

VII. ¿Está de acuerdo que dentro en la Institución Educativa se genera ruido?

1. Totalmente en desacuerdo

2. En desacuerdo

3. Indiferente (ni en acuerdo ni en desacuerdo)

4. De acuerdo

5. Totalmente de acuerdo

VIII. ¿Está de acuerdo en que el ruido es generado por las personas en el desarrollo de sus actividades cotidianas?

1. Totalmente en desacuerdo

2. En desacuerdo

3. Indiferente (ni en acuerdo ni en desacuerdo)

4. De acuerdo

5. Totalmente de acuerdo

IX. ¿Está usted de acuerdo en que la exposición prolongada al ruido puede generar daños en la salud de las personas?

1. Totalmente en desacuerdo

2. En desacuerdo

3. Indiferente (ni en acuerdo ni en desacuerdo)
4. De acuerdo

5. Totalmente de acuerdo

X. ¿Qué nivel de conocimiento tiene sobre el daño que puede causar el ruido en la salud las personas?

1. Muy bajo

2. Bajo

3. Medio

4. Alto

5. Muy Alto

XI. ¿Está usted de acuerdo en que el ruido puede afectar el rendimiento académico de los estudiantes?

1. Totalmente en desacuerdo

2. En desacuerdo

3. Indiferente (ni en acuerdo ni en desacuerdo)

4. De acuerdo

5. Totalmente de acuerdo

XII. ¿Está de acuerdo en que es importante generar campañas escolares que ayuden a prevenir y disminuir el ruido en la Institución?

1. Totalmente en desacuerdo

2. En desacuerdo

3. Indiferente (ni en acuerdo ni en desacuerdo)

4. De acuerdo

5. Totalmente de acuerdo

Gracias por su colaboración 